\title{
Frequency and damping identification in flutter flight testing using Singular Value Decomposition (SVD) and QR factorization
}

\author{
José Barros-Rodríguez ${ }^{+}$, Roberto Flores Le Roux ${ }^{\#}$, Jesús López-Díez* and Rodrigo Martinez-Val ${ }^{\triangleleft} *$ \\ + Logistic Center of Ordnance and Experimentation (CLAEX), Spanish Air Force (SAF) \\ \# CIMNE - Centro Internacional de Métodos Numéricos en Ingeniería, Barcelona, Spain \\ 4 Departamento de Vehículos Aeroespaciales, Universidad Politécnica de Madrid, Madrid, Spain \\ * Corresponding author. Tel.: +34-913366367.E-mail address: rodrigo.martinezval@upm.es
}

\begin{abstract}
A new method, based on Singular Value Decomposition (SVD) and QR factorization, has been developed and applied to the analysis of F-18 flutter flight-test data. The method is capable of identifying the frequency and damping of the critical aircraft modes, those responsible for the flutter phenomenon. The procedure relies on the capability of SVD for the analysis, modelling and prediction of data series with periodic features and also on its power to identify matrix rank. The analysis of simulated and real flutter flight test data demonstrates the effectiveness, robustness, noise-immunity and the capability for automation of the method proposed under specific conditions.
\end{abstract}

Keywords: flight flutter; damping; modal parameters identification; SVD; QR factorization

\section{Background}

Flutter flight-testing remains a demanding task, mainly due to safety concerns. Great efforts are also required in several related areas, including flight data analysis.

A flutter test-campaign was completed on an F-18 aircraft configured with a wingtip-mounted IRIS$\mathrm{T}$ Missile and several underwing stores in order to establish the boundaries of flight envelope. Two types of excitations are employed in F-18 flutter flight-testing. Swept-frequency aileron deflection excitation is used to identify structural modal parameters. This provides an estimation of the frequency for each mode but yields low-accuracy results for damping. Dwell (3 seconds fixed frequency) aileron deflection excitation is used to obtain a better estimation of damping and to identify non-linear effects, giving an indication of energy exchange between critical modes. From the free response (which lasts for 2 or 3 seconds) obtained after dwell excitation, damping and frequency information can be extracted.

Free response signals from dwell excitation are short as well as scarce since, unlike ground tests, flight time is very costly and tests are seldom repeated. Moreover, as the stability boundary is approached, the spacing between the critical modes is reduced (frequency coalescence), their energy difference increases (larger damping difference) and the nonlinearities become more pronounced (amplitude increases). In summary, close to the stability border, where information on critical modes (those leading to flutter phenomena) is of vital importance, the measurements required become very problematic. 
These facts make modal identification in flutter testing especially difficult. To overcome these problems several methodologies have been studied. The Morlet wavelet has been employed combined with several methods to avoid end-effects for short signals; Kijewski \&Kareem ${ }^{1,2}$ use signal padding to extend the signal while Slavic \& Boltezar ${ }^{3-5}$ apply several methods without signal extension, modifying instead the mother wavelet. Brenner ${ }^{6}$ employs first only wavelet and later combines Wavelet and $\mathrm{SVD}^{7}$ (Singular Value Decomposition) to analyze flight test data while ThaiHoa \& Kamura $^{8}$ combines wavelet with FDD (Frequency Domain Decomposition). Brenner \& Prazenica ${ }^{9}$ investigate the application of Hilbert- Huang Transformation for the analysis of aeroelastic flight data. Kijewski \&Kareem ${ }^{10}$ employ Random Decrement Technique combined with bootstrap statistics to estimate damping while Rodrigues and Brincker ${ }^{11}$ study its use combined with other modal methods to unveil the modal parameters of short signals. Juang and Pappa ${ }^{18}$ develop the ERA (Eigenvalue Realization Algorithm), further explained in Gawronski's Book ${ }^{19}$.

Short free-response data samples from the IRIS-T test campaign were analysed with a SVD and QR factorization based method which is presented in section 3. The method can be employed even when weak nonlinear effects are present, as is the case for an F-18 with heavy loads in outboard wing stations. In section 4 the performance of the method is demonstrated with synthetic as well as real flight-tests data.

\section{Characteristic features of SVD}

Given any $m \times n$ real matrix A, there exist a $m \times m$ real orthogonal matrix $\mathbf{U}$, a $n \times n$ real orthogonal matrix $\mathbf{V}$ and a $m \times n$ rectangular diagonal matrix $\mathbf{S}$, such that ${ }^{12-16}$ :

$$
\begin{array}{ll}
\mathbf{A}=\mathbf{U S V}^{\mathbf{T}} & \mathbf{S}=\mathbf{U}^{\mathbf{T}} \mathbf{A} \mathbf{V} \\
\mathbf{V}^{\mathbf{T}} \mathbf{V}=\mathbf{I} & \mathbf{U}^{\mathrm{T}} \mathbf{U}=\mathbf{I} \quad \text { because } \mathbf{U} \text { and } \mathbf{V} \text { are orthogonal }
\end{array}
$$

The elements of $\mathbf{S}$, called singular values, are arranged in a non-increasing order (that is, for $m<n)$ :

$$
\mathbf{S}=\left[\begin{array}{ccccccc}
s_{1} & 0 & . & 0 & 0 & . & 0 \\
0 & s_{2} & . & 0 & 0 & . & 0 \\
. & . & . & . & . & . & . \\
0 & 0 & . & s_{m} & 0 & . & 0
\end{array}\right] \quad \text { if } \operatorname{rank} \mathbf{A}=m \quad \begin{aligned}
& s_{1} \geq s_{2} \geq \ldots \geq s_{m}>0 \\
& \text { if } \operatorname{rank} \mathbf{A}=r<m
\end{aligned} \quad\left\{\begin{array}{c}
s_{1} \geq s_{2} \geq \ldots \geq s_{r}>0 \\
s_{r+1}=\ldots=s_{m}=0
\end{array}\right.
$$

It can be concluded that the SVD decomposition indicates the effective rank of the matrix $\mathbf{A}$.

Using the Frobenius norm ${ }^{12}$, an energy balance can be established between the values of $\mathbf{A}$ and the singular values:

$$
\sum_{1}^{m} \sum_{1}^{n}\left(a_{i j}\right)^{2}=\|A\|_{F}^{2}=s_{1}^{2}+s_{2}^{2}+\ldots .+s_{m}^{2} \quad \text { where } a_{i j} \text { are the elements of } \mathbf{A}
$$

The SVD decomposition is very stable and robust ${ }^{12}$ since perturbations in the elements of $\mathbf{A}$ give rise to fluctuations of the same or smaller order in the singular values.

In summary, since SVD gives the rank of $\mathbf{A}$, it provides the number of rows that are linearly independent. And because SVD remains stable if the elements of $\mathbf{A}$ are contaminated with noise, it is still able to identify the number of linearly independent rows. 
In order to identify a base of the space spanned by the rows of $\mathbf{A}$, which consists of $r$ vectors $\mathbf{a}_{\mathbf{i}}(\mathrm{r}$ being the rank of $\mathbf{A}$ ), a subset selection criterion as proposed by Prof. Kanjilal ${ }^{12}$ is needed. To this effect, the row having the maximum Euclidean norm is chosen first. Next, the row having maximum orthogonal component to the row just selected is chosen. The process is repeated until $r$ rows have been selected.

\section{Proposed method for system identification}

\subsection{Hypothesis of linearity}

The basic hypothesis used in this method is that there is a structural equivalent system in which the structural modes can be represented by harmonic signals exponentially damped ${ }^{17}$.

This assumption implies the existence of a standard set of linearized equations of motion:

$$
\mathbf{M} \ddot{\mathbf{x}}+\mathbf{C} \dot{\mathbf{x}}+\mathbf{K x}=0
$$

where $\mathbf{M}, \mathbf{C}$ and $\mathbf{K}$ denote the mass, damping and stiffness matrices respectively and $\mathbf{x}$ is the displacement vector. The solution of Eq. 4 can be expressed as a function of the modal coordinates as:

$$
\mathbf{x}=\boldsymbol{\Phi} \mathbf{q}(t)=\sum_{r=1}^{n} \boldsymbol{\varphi}^{\mathbf{r}} q^{r}(t)
$$

In Eq. $5 \Phi$ denotes the modal matrix whose rows are the modal shape vectors $\boldsymbol{\varphi}^{r}$. The vector $\mathbf{q}$ contains the values of the modal coordinates $\mathrm{q}^{\mathrm{r}}$. The time evolution of the modal coordinates is given by:

$$
q^{r}(t)=e^{-\xi^{r} 2 \pi f_{n}^{r} t} \sin \left(2 \pi f_{d}^{r} t+\phi^{r}\right) \quad f_{d}^{r}=f_{n}^{r} \sqrt{1-\xi^{r 2}}
$$

$f_{d}^{r}$ denotes the damped frequency of mode $\mathrm{r}$, whose natural frequency and damping ratio are $f_{n}^{r}$ and $\xi^{r}$; on its side, $\phi^{r}$ is the phase of the mode r.

A sensor of the aircraft is able to measure a component $x_{i}(t)$ of the vector of displacements $x(t)$. Assuming that the free response comprises only exponentially-damped harmonic signals, each one with constant frequency and a damping coefficient, the following expression can be obtained:

$$
x_{i}(t)=\sum_{r=1}^{n} \lambda_{i}^{r} e^{-\xi^{r} 2 \pi f_{n}^{r} t} \sin \left(2 \pi f_{d}^{r} t+\phi^{r}\right)
$$

However, the signal form a sensor of the aircraft will also contain electrical noise, forced response to turbulence and the effect on nonlinearities.

$$
\tilde{x}_{i}(t)=\sum_{r=1}^{n} \lambda_{i}^{r} e^{-\xi^{r} 2 \pi f_{n}^{r} t} \sin \left(2 \pi f_{d}^{r} t+\phi^{r}\right)+\text { noise }+ \text { turbulence response }+ \text { nonlinearity }
$$

If the noise and response to turbulence are random and Gaussian distributed and only weak nonlinear effects are present, the method proposed hereafter is applicable. The method also provides an assessment of the importance of non-linear effects. 


\subsection{Matrix assembly with a single sensor and dependence between rows}

In the case where there is only one sensor, in principle, all the modes of the system could be identified. If, however, the sensor is placed close to a node, the corresponding mode cannot be detected because the signal it creates is too weak (i.e. the relevant component of the modal vector is small).

Let us take a signal of length $T$ recorded with sampling frequency $f_{s}$ and containing an odd number of samples (for the sake of convenience).

$$
\tilde{x}(t) \stackrel{\text { SAMPLING }}{\rightarrow} \tilde{\mathbf{x}}_{\mathbf{2 N}}=\left[\begin{array}{llll}
\tilde{x}(0) & \tilde{x}\left(T_{s}\right) & \tilde{x}\left(2 T_{s}\right) & \tilde{x}\left(3 T_{s}\right) \ldots . . \tilde{x}\left(2 N T_{s}\right)
\end{array}\right]
$$

The time between samples is given by $T_{s}=1 / f_{s}$ and the total number of sample points is $2 \mathrm{~N}+1=\mathrm{T}_{\mathrm{s}} / \mathrm{T}_{\mathrm{s}}+1$. The first step of the method is to build a symmetric square matrix $\mathbf{A}$. This is achieved taking as the first row the first $\mathrm{N}+1$ samples of the signal, the second row contains the signal offset by one record, the third row is the signal offset by 2 records and so on:

$$
\mathbf{A}=\left[\begin{array}{ccclc}
\tilde{x}(0) & \tilde{x}\left(T_{s}\right) & \tilde{x}\left(2 T_{s}\right) & \cdot & \tilde{x}\left(N T_{s}\right) \\
\tilde{x}\left(T_{s}\right) & \tilde{x}\left(2 T_{s}\right) & \tilde{x}\left(3 T_{s}\right) & \cdot & \tilde{x}\left((N+1) T_{s}\right) \\
\tilde{x}\left(2 T_{s}\right) & \tilde{x}\left(3 T_{s}\right) & \tilde{x}\left(3 T_{s}\right) & \cdot & \tilde{x}\left((N+2) T_{s}\right) \\
\cdot & \cdot & \cdot & \cdot & \cdot \\
\tilde{x}\left(N T_{s}\right) & \tilde{x}\left((N+1) T_{s}\right) & \tilde{x}\left((N+2) T_{s}\right) & \cdot & \tilde{x}\left(2 N T_{s}\right)
\end{array}\right]
$$

Let us now study the relationship between the different rows of the matrix. It is assumed that there are $\mathrm{n}$ modes and that the noise, turbulence and nonlinearity can be modelled as a white Gaussian noise $\varepsilon$.

Note that in the following the subindex I corresponding to the sensor has been removed.

$$
\begin{aligned}
& \tilde{x}^{r}(t)=\lambda^{r} e^{-\xi^{r} 2 \pi f_{n}^{r} t} \sin \phi \cos \left(2 \pi f_{d}^{r} t\right)+\lambda^{r} e^{-\xi^{r} 2 \pi f_{n}^{r} t} \cos \phi \sin \left(2 \pi f_{d}^{r} t\right)+\varepsilon(t) \quad \tilde{x}(t)=\sum_{r=1}^{n} \tilde{x}^{r}(t) \\
& \tilde{\mathbf{x}}^{\mathrm{r}}(N)=\left[\tilde{x}^{r}(0) \tilde{x}^{r}\left(T_{s}\right) \tilde{x}^{r}\left(2 T_{s}\right) \cdots \tilde{x}^{r}\left(N T_{s}\right)\right] \quad \tilde{\mathbf{x}}(N)=\sum_{r=1}^{n} \tilde{\mathbf{x}}^{\mathrm{r}}(N) \\
& \tilde{\mathbf{x}}^{\mathrm{r}}(N+\alpha)=\left[\tilde{x}^{r}\left(\alpha T_{s}\right) \tilde{x}^{r}\left((\alpha+1) T_{s}\right) \tilde{x}^{r}\left((\alpha+2) T_{s}\right) \cdots \tilde{x}^{r}\left((N+\alpha) T_{s}\right)\right]
\end{aligned}
$$

Each mode has been split into its cosine and sine components in order to remove the phase from the argument of the trigonometric functions.

$$
\begin{array}{lll}
\tilde{x}^{r}(t)=a^{r} c^{r}(t)+b^{r} s^{r}(t)+\varepsilon(t) & \\
a^{r}=\lambda^{r} \sin \phi & c^{r}(t)=e^{-\xi^{r} 2 \pi f_{n}^{r} t} \cos \left(2 \pi f_{d}^{r} t\right) & \mathbf{c}^{\mathrm{r}}(N)=\left[c^{r}(0) c^{r}\left(T_{s}\right) c^{r}\left(2 T_{s}\right) \cdots c^{r}\left(N T_{s}\right)\right] \\
b^{r}=\lambda^{r} \cos \phi & s^{r}(t)=e^{-\xi^{r} 2 \pi f_{n}^{r} t} \sin \left(2 \pi f_{d}^{r} t\right) & \mathbf{s}^{\mathrm{r}}(N)=\left[s^{r}(0) s^{r}\left(T_{s}\right) s^{r}\left(2 T_{s}\right) \cdots s^{r}\left(N T_{s}\right)\right] \\
\tilde{\mathbf{x}}^{\mathrm{r}}(N)=a^{r} \mathbf{c}^{\mathrm{r}}(N)+b^{r} \mathbf{s}^{\mathrm{r}}(N)+\varepsilon &
\end{array}
$$


For any natural number $\alpha$ :

$$
\begin{aligned}
& a^{r} \mathbf{c}^{\mathbf{r}}(N+\alpha)+b^{r} \mathbf{s}^{\mathbf{r}}(N+\alpha)= \\
& e^{-\xi^{r} 2 \pi f_{n}^{r} \alpha T_{s}}\left[\left(a^{r} \cos \left(2 \pi f_{d}^{r} \alpha T_{s}\right)+b^{r} \sin \left(2 \pi f_{d}^{r} \alpha T_{s}\right)\right) \mathbf{c}^{\mathbf{r}}(N)+\left(b^{r} \cos \left(2 \pi f_{d}^{r} \alpha T_{s}\right)-a^{r} \sin \left(2 \pi f_{d}^{r} \alpha T_{s}\right)\right) \mathbf{s}^{\mathbf{r}}(N)\right]
\end{aligned}
$$

The different rows of matrix $\mathbf{A}$ are obtained by delaying the first row by an integer number of records. In light of Eq. 10, for a linear and free of noise, the rank of matrix $\mathbf{A}$ (the number of nonzero singular values on its SVD) should match the number of independent vectors $\mathbf{c}^{\mathbf{r}}$ and $\mathbf{s}^{\mathbf{r}}$. That is, the rank of $\mathbf{A}$ would equal twice the number of modes. On the other hand, in a real system with noise, turbulence and weak nonlinearity all the singular values are nonzero. The smallest singular values appear due to these additional factors.

$$
\mathbf{A}=\mathbf{U S V}^{\mathbf{T}} / \mathbf{S}=\left[\begin{array}{cccc}
S_{1} & & & 0 \\
& S_{2} & & \\
& & \ddots & \\
0 & & & S_{N+1}
\end{array}\right] S_{1} \geq S_{2} \geq \cdots \geq S_{2 n}>S_{2 n+1} \geq \cdots \geq S_{N+1} \geq 0
$$

If the conditions under which the method is applicable are met, there will be $2 \mathrm{n}$ dominant singular values with the rest being considerably smaller. This provides a direct measure of the modal order of the system. If there is no clear distinction between both sets of singular values this means the method is not applicable (e.g. there are strong nonlinearities in the response). By dropping the small singular values it is possible to filter out most of the noise. This is achieved by building the matrix $\mathbf{A}_{\mathbf{C}}$ which is almost noise-free, in which small singular values have being equalled to zero:

$$
\mathbf{A}_{\mathbf{C}}=\mathbf{U S}_{\mathbf{C}} \mathbf{V}^{\mathbf{T}} \Leftrightarrow \mathbf{S}_{\mathbf{C}}=\left[\begin{array}{llll}
S_{1} & & & 0 \\
& S_{2} & & \\
& & \ddots & \\
0 & & & 0
\end{array}\right] \quad \begin{array}{lll}
S_{C_{i}}=S_{i} & i=1, \ldots, 2 n \\
S_{C_{j}}=0 & j=2 n+1, \ldots, N+1
\end{array}
$$

In order to obtain the noise-free signal the first and last columns of matrix $\mathbf{A}_{\mathbf{C}}$ must be combined

$$
\mathbf{A}_{\mathbf{C}}=\left[\begin{array}{cccc}
x_{C}(0) & x_{C}\left(T_{s}\right) & \cdot & x_{C}\left(N T_{s}\right) \\
x_{C}\left(T_{s}\right) & x_{C}\left(2 T_{s}\right) & \cdot & x_{C}\left((N+1) T_{s}\right) \\
x_{C}\left(2 T_{s}\right) & x_{C}\left(3 T_{s}\right) & \cdot & x_{C}\left((N+2) T_{s}\right) \\
\cdot & \cdot & \cdot & \cdot \\
x_{C}\left(N T_{s}\right) & x_{C}\left((N+1) T_{s}\right) & \cdot & x_{C}\left(2 N T_{s}\right)
\end{array}\right] \rightarrow \mathbf{x}_{\mathbf{C}}(2 N)=\left[x_{C}(0) x_{C}\left(T_{s}\right) \ldots x_{C}\left(2 N T_{s}\right)\right]
$$

Several existing methods, such as ERA ${ }^{18,19}$ and $\mathrm{CVA}^{20,21}$ build up a matrix A (Hankel matrix) and employ SVD for model reduction, justification based on linear dependency between matrix's rows when the signals can be approximated by exponentially damped harmonic functions is presented in this paper. 


\subsection{QR factorization and column pivoting}

Any set of $2 n$ columns from matrix $\mathbf{A}_{\mathbf{C}}$ would form a base equivalent to the $2 n$ vectors $\mathbf{c}^{\mathbf{r}}$ and $\mathbf{s}^{\mathbf{r}}$. It is important, however, to choose the most adequate vectors. These are the vectors that are the most orthogonal to each other and have maximum norm. An efficient means of achieving this goal is $\mathrm{QR}^{12,15}$ factorization with column pivoting. A column pivoting strategy is used to obtain a permutation matrix $\mathbf{E}$ such that:

$$
\mathbf{A}_{\mathbf{C}} \mathbf{E}=\mathbf{Q R} \quad / \quad \mathbf{Q Q}^{\mathbf{T}}=\mathbf{I} \quad, R_{i j}=0 \forall i>j \quad \rightarrow \quad \mathbf{B}=\mathbf{E}^{\mathbf{T}} \mathbf{A}_{\mathbf{C}}
$$

The matrix $\mathbf{B}$ is ordered in such a way that its first $2 \mathrm{n}$ rows are the best base available to represent the space spanned by the vectors $\mathbf{c}^{\mathbf{r}}$ and $\mathbf{s}^{\mathbf{r}}$. Therefore, it is enough to keep these $2 \mathrm{n}$ rows and the rest can be discarded. A truncated matrix $\mathbf{B}_{\mathbf{t}}$ can be built keeping only the first $2 \mathrm{n}$ rows:

$$
\left.\mathbf{B}_{\mathbf{t}}=\left[\begin{array}{cccc}
x\left(\eta T_{s}\right) & x\left((\eta+1) T_{s}\right) & \cdot & x\left((\eta+N) T_{s}\right) \\
x\left(v T_{s}\right) & x\left((v+1) T_{s}\right) & \cdot & x\left((v+N) T_{s}\right) \\
\cdot & \cdot & \cdot & \cdot \\
x\left(\sigma T_{s}\right) & x\left((\sigma+1) T_{s}\right) & \cdot & x\left((\sigma+N) T_{s}\right)
\end{array}\right]\right\} 2 n \text { rows }
$$

Since matrix $\mathbf{B}_{\mathbf{t}}$ is of rank $2 n$, its SVD has a singular matrix $\mathbf{S}(2 n$ by $\mathrm{N}+1)$ with $2 n$ nonzero singular values.

Column pivoting ${ }^{12}$ based on $\mathrm{QR}$ decomposition in order to optimize row selection in matrix $\mathbf{A}$ is not employed in other modal analysis methods and is inspired by Prof. Kanjilal ${ }^{12}$ subset selection.

\subsection{Frequency and damping determination}

An augmented matrix $\mathbf{B}^{*}$ can be obtained adding an additional row to $\mathbf{B}_{\mathbf{t}}$. If the new row is linearly independent from the old ones (i.e. it is linearly independent from the $2 n$ vectors $\mathbf{c}^{\mathbf{r}}$ and $\mathbf{s}^{\mathbf{r}}$ ) $\mathbf{B}^{*}$ will be of rank $2 n+1$. On the other hand, if the new vector is a linear combination of the rows of $\mathbf{B}_{t}$, the ranks of $\mathbf{B}_{\mathbf{t}}$ and $\mathbf{B}^{*}$ shall remain equal due to the additional singular value being null.

In order to find the modes contained in the signal a parametric family of exponentially-damped sinusoidal functions (normalized with their Euclidean norm) is created. The free parameters are the frequency and the reduced damping $\left(f_{K}, \xi_{K}\right)$.

$$
y_{K}(t)=e^{-\xi_{K} 2 \pi f_{K} t} \sin \left(2 \pi f_{K} t\right) \Rightarrow \mathbf{Y}_{\mathrm{K}}(N)=\left[y_{K}(0) y_{K}\left(T_{s}\right) y_{K}\left(2 T_{s}\right) \cdots y_{K}\left(N T_{s}\right)\right] \Rightarrow \mathbf{Y}_{\mathrm{K}}^{\mathbf{1}}=\frac{\mathbf{Y}_{\mathrm{K}}}{\left\|\mathbf{Y}_{\mathrm{K}}\right\|}
$$

The $\mathbf{Y}_{\mathrm{K}}^{1}$ vectors are used to build a family of augmented matrices $\mathbf{B}_{\mathrm{K}}^{*}$. The SVD transformation is then applied to each member of this family: 


$$
\begin{aligned}
& \left.f_{K} \rightarrow y_{K}(t) \rightarrow \mathbf{B}_{\mathrm{K}}^{*}=\left[\begin{array}{cccc}
x\left(\eta T_{s}\right) & x\left((\eta+1) T_{s}\right) & \cdot & x\left((\eta+1) T_{s}\right) \\
x\left(v T_{s}\right) & x\left((v+1) T_{s}\right) & \cdot & x\left((v+N) T_{s}\right) \\
\cdot & \cdot & \cdot & \cdot \\
x\left(\sigma T_{s}\right) & x\left((\sigma+1) T_{s}\right) & \cdot & x\left((\sigma+N) T_{s}\right) \\
y_{K}^{1}(0) & y_{K}^{1}\left(T_{s}\right) & \cdot & y_{K}^{1}\left(N T_{s}\right)
\end{array}\right]\right\} 2 n+1 \text { rows } \\
& \stackrel{S V D}{\rightarrow} \mathbf{B}_{\mathrm{K}}^{*}=\mathbf{U}_{\mathbf{K}} \mathbf{S}_{\mathbf{K}} \mathbf{V}_{\mathbf{K}}^{\mathbf{T}}
\end{aligned}
$$

To evaluate the rank of $\mathbf{B}_{K}^{*}$ the product of the $2 n+1$ singular values of $\mathbf{S}_{\mathbf{K}}$ is taken. Let us denote it by $\Psi_{\mathrm{K}}$.

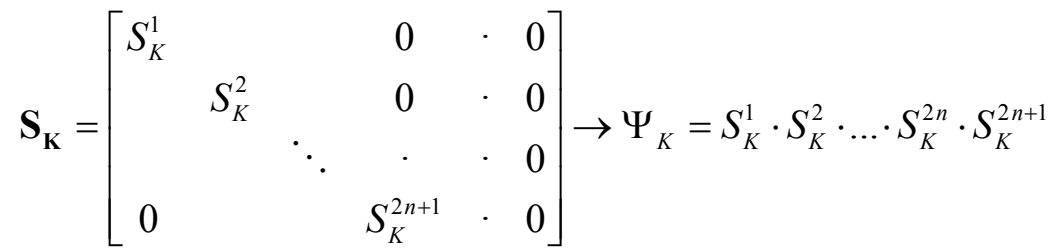

Note that $\Psi_{K}$ is actually the determinant of the reduced matrix obtained retaining only the first $2 n+1$ columns of $\mathbf{S}_{\mathbf{K}}$. This determinant vanishes if matrix $\mathbf{B}_{\mathrm{K}}^{*}$ is of rank 2n. For each pair of parameters $\left(f_{\mathrm{K}}, \xi_{\mathrm{K}}\right)$ a $\Psi_{\mathrm{K}}$ value can be computed which assesses to what degree the new vector $\mathbf{Y}_{\mathrm{K}}^{1}$ is independent from the $\left(\mathbf{c}^{\mathbf{r}}, \mathbf{s}^{\mathbf{r}}\right)$ base. Those $\mathbf{Y}_{\mathrm{K}}^{\mathbf{1}}$ vectors which more closely match the modes of the system yield minimum values of $\Psi_{K}$.

In order to obtain a comprehensive coverage a sequence of $\left(f_{K}, \xi_{K}\right)$ pairs must be built which spans the range of interest with an adequate resolution. To simplify the interpretation of the results it is desirable to build a sequence in which consecutive elements correspond to monotonically increasing damping ratios for a given frequency value:

$\begin{array}{ccc}K & f_{K} & \xi_{K} \\ 1 & f_{0} & \xi_{0} \\ 2 & f_{0} & \xi_{0}+\Delta \xi \\ \vdots & \vdots & \vdots \\ \beta+1 & f_{0} & \xi_{0}+\beta \Delta \xi \\ \beta+2 & f_{0}+\Delta f & \xi_{0} \\ \beta+3 & f_{0}+\Delta f & \xi_{0}+2 \Delta \xi \\ \vdots & \vdots & \vdots \\ 2 \beta+2 & f_{0}+\Delta f & \xi_{0}+\beta \Delta \xi \\ 2 \beta+3 & f_{0}+2 \Delta f & \xi_{0} \\ \vdots & \vdots & \vdots\end{array}$


While SVD is a very robust algorithm for matrix analysis, it cannot always avoid spurious matches between the $\mathbf{Y}_{\mathrm{K}}^{\mathbf{1}}$ vectors and noise present in the signal. Nevertheless, it must be noted that the analytical $\mathbf{Y}_{\mathrm{K}}^{\mathbf{1}}$ vectors were built using the sine function, but could have been built from the cosine function as well. When the mode identified is real the values of $\Psi_{\mathrm{K}}$ for $\mathbf{Y}_{\mathrm{K}}^{\mathbf{1}}$ vectors built with both sine and cosine functions must be local minima. Failure to meet this condition indicates that the mode identified is spurious and must be discarded.

Current method employs SVD to identify frequency and damping inspired in other technological areas as image correlation while other modal analysis techniques prefer Least Square algorithms

\subsection{Method for multiple sensors}

When there are $\mathrm{S}$ different sensors, the first step is to normalize each signal using its Euclidean norm. Once this has been done, the algorithm closely follows the single-sensor case:

$$
\begin{aligned}
& \tilde{x}_{k}(t) \stackrel{\text { SAMPLING }}{\rightarrow} \tilde{\mathbf{x}}_{\mathbf{k}}(2 N)=\left[\begin{array}{lllll}
\tilde{x}_{k}(0) & \tilde{x}_{k}\left(T_{s}\right) & \tilde{x}_{k}\left(2 T_{s}\right) & \tilde{x}_{k}\left(3 T_{s}\right) \ldots . . \tilde{x}_{k}\left(2 N T_{s}\right)
\end{array}\right] \quad k=1,2, \cdots, S \\
& \tilde{\mathbf{x}}_{\mathbf{k}}(\alpha, \beta)=\left[\begin{array}{lllll}
\tilde{x}_{k}\left(\alpha T_{s}\right) & \tilde{x}_{k}\left((\alpha+1) T_{s}\right) & \cdots & \tilde{x}_{k}\left((\beta-1) T_{s}\right) & \tilde{x}_{k}\left(\beta T_{s}\right)
\end{array}\right] \quad \alpha, \beta \in \square
\end{aligned}
$$

In this case the square matrix $\mathbf{A}$ contains samples from all sensors:

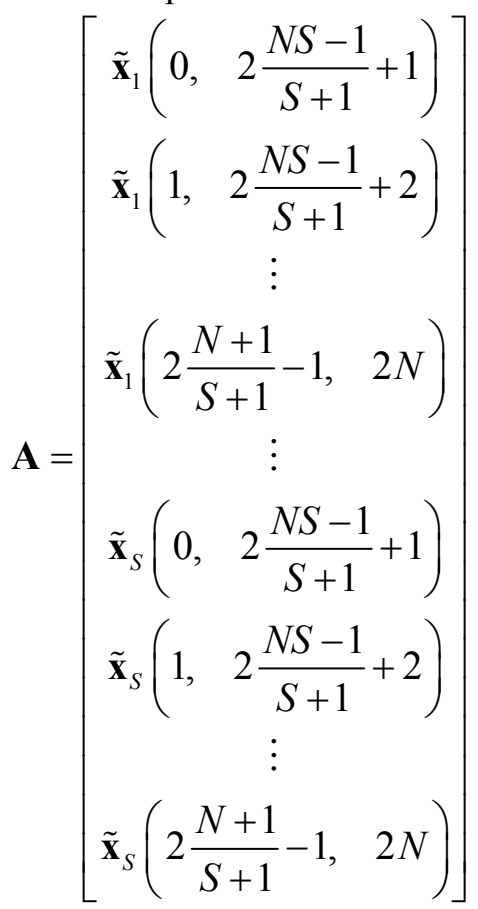

Next, the SVD is applied to $\mathbf{A}$ and reduced singular value matrix $\mathbf{S}_{\mathbf{C}}$ is formed in order to remove unwanted noise. The filtered signal can be recovered from $\mathbf{A}_{\mathbf{C}}$ matrix: 


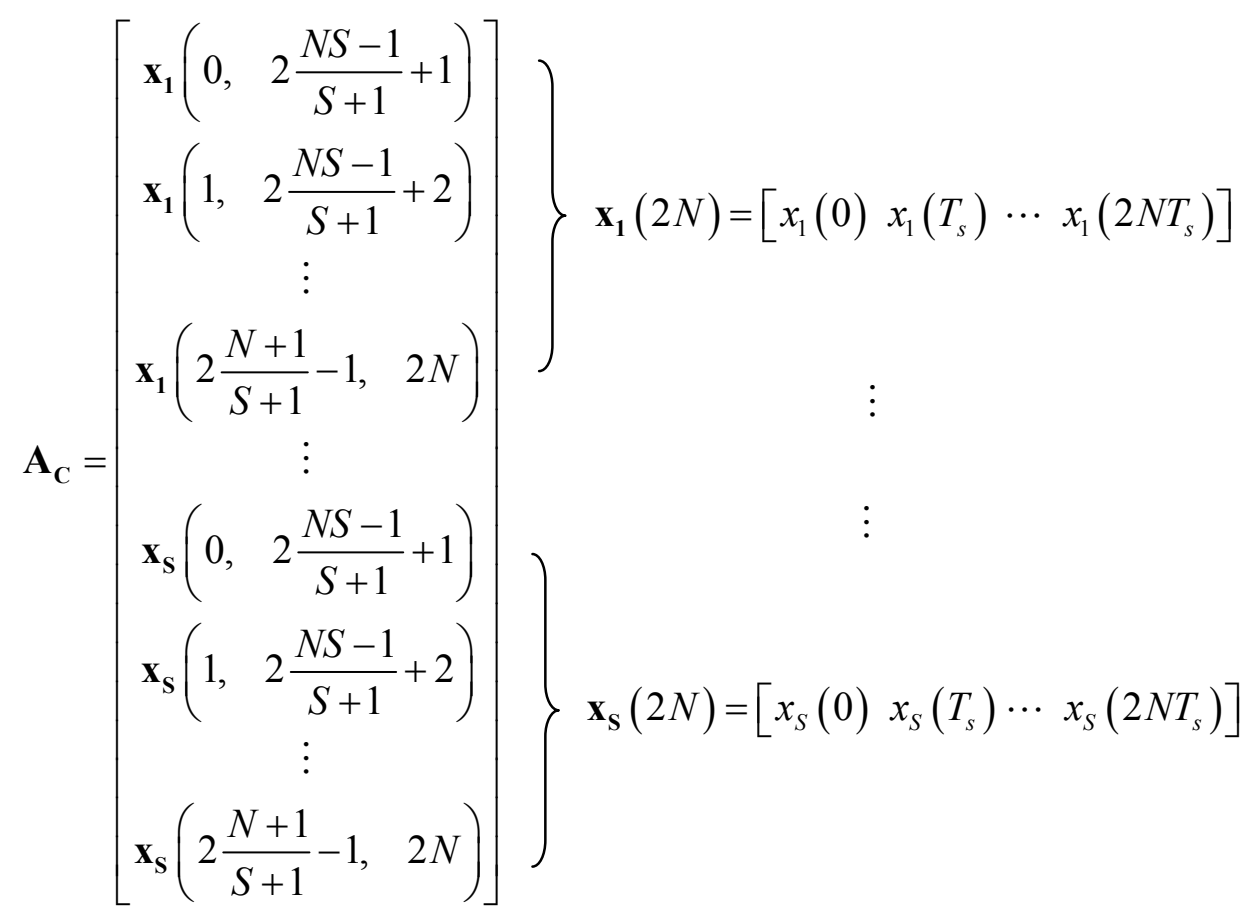

The rest of the steps to follow are identical to single-sensor procedure.

\section{RESULTS AND DISCUSSION}

\subsection{System Identification with Synthetic Data}

Tests were conducted using the basic MATLAB 6.5 software without specialized toolboxes, applying the method to exponentially-damped harmonic functions to which Gaussian noise was superimposed. An example is shown in Eq. 26 with two harmonic functions whose parameters are similar to the typical modal parameters found in the real tests. The frequencies of the functions are $5.4 \mathrm{~Hz}$ and $6.0 \mathrm{~Hz}$. The amplitudes are respectively 1.0 and 0.5 and the damping coefficients 0.015 and 0.03 . There is a variable phase shift between the two functions. The signal was intentionally contaminated with various levels (relative to the $6.0 \mathrm{~Hz}$ component) of Gaussian noise. Sampling frequency is $500 \mathrm{~Hz}$, equal to that available in the real tests presented later:

$$
y(t)=e^{-2 \pi 0.015 \cdot 5.4 t} \sin (2 \pi 5.4 t)+\frac{1}{2} e^{-2 \pi 0.03 \cdot 6.0 t} \sin (2 \pi 6.0 t+\varphi)+\varepsilon_{N(0,0.5)}
$$

The results are shown in Table 1.

It can be observed that results are not influenced by the phase shift due to the nature of the method. In the case when noise is $8 \mathrm{~dB}$ and phase is $15^{\circ}$ Table 1 depicts both the original signal and the filtered data obtained using the SVD-based method and retaining 4 singular values. 
Table 1 - Frequency $(\mathrm{Hz})$ and damping coefficients for various values of phase and noise

\begin{tabular}{|c|c|c|c|c|c|c|c|}
\hline & \multicolumn{6}{|c|}{ Phase } \\
\hline & & 0 & 15 & 30 & 45 & 60 & 75 \\
\hline \multirow{3}{*}{ Noise Level } & 8 & \multicolumn{6}{|c|}{$\begin{array}{l}\mathrm{f}=5.4 \text { and } \xi=0.015 \\
\mathrm{f}=6.0 \text { and } \xi=0.025\end{array}$} \\
\hline & 6 & \multicolumn{6}{|c|}{$\begin{array}{c}f=5.4 \text { and } \xi=0.015 \\
f=6.0 \text { and error in damping, with a low probability of missing the mode }\end{array}$} \\
\hline & 2 & \multicolumn{6}{|c|}{$\begin{array}{r}f=5.4 \text { and } \xi=0.001-0.002 \\
f=6.0 \text { and error in damping, with a high probability of missing the mode }\end{array}$} \\
\hline
\end{tabular}

Once the noise has been removed from the signal, the modal identification step can be performed. In the case when noise is $8 \mathrm{~dB}$ and phase is $15^{\circ}$ Table 2 shows the evolution of the $\Psi$ parameter, as different combinations of frequency and damping are tested. Each curve segment corresponds to a single frequency value, which is labelled on the horizontal axis. The frequency difference between adjacent curves $(\Delta \mathrm{f})$ is $0.2 \mathrm{~Hz}$ while the jump in damping ratio between consecutive points $(\Delta \xi)$ is 0.005 .

The curve shows two distinct peaks at $\mathrm{f}=5.4 \mathrm{~Hz}, \xi=0.015$ and $\mathrm{f}=6.0 \mathrm{~Hz}, \xi=0.025$. The frequencies have been correctly estimated. Furthermore, for a $2 \mathrm{~s}$ sample length conventional frequency-domain analysis techniques would be at the limit of their resolving power $(0.5 \mathrm{~Hz})$ making it extremely difficult to identify the two modes due the high noise level. The method proposed compares favourably in this respect. Regarding the damping ratio, a reasonable estimate has been obtained. It must be stressed that high noise content is especially troublesome in this respect, so the result is actually fairly accurate.

\subsection{System Identification with Flight Data}

The method has been employed on signals from sensors embedded on the wing of the EF-18 fighter aircraft obtained during an IRIS-T missile integration flutter test-flight. Although it has been used only for post-processing of the signals on ground, the short computing time (around 1 second) with MATLAB software indicates that the method could be working combined with prediction algorithms (Flutter Margin) to assist in the decision whether or not to proceed to a more critical points during in-flight testing. Aircraft was configured with wingtip-mounted missile and several heavy under-wing stores. Test point was performed fairly close to the final stability border and corresponds to an asymmetric dwell excitation at $4.5 \mathrm{~Hz}$. The free response unprocessed signals resulting from such excitation are shown in Table 3, while the frequency spectrum of the signals as well as the comparison between processed and unprocessed LM13 signal have been included in Figure 4. Given that data from multiple acquisition channels is available both the single-sensor and the multiple-sensor variants of the method can be put to test and their results can be compared. Data was collected for two seconds using a $500 \mathrm{~Hz}$ sampling frequency.

The signals were passed through an analog band-pass filter on the aircraft to remove components below $1 \mathrm{~Hz}$ and over $20 \mathrm{~Hz}$. The method for single sensor has been applied to each individual signal while the method for multiple sensors has been used with the complete set. The results of the analysis are summarized in Table 2. 
Table 2- Frequency $(\mathrm{Hz})$ and damping coefficients obtained with SVD-based analysis method

\begin{tabular}{|c|c|c|c|c|c|c|c|}
\cline { 2 - 8 } \multicolumn{1}{c|}{} & \multicolumn{6}{c|}{ SVD Method with single sensor } & SVD Method with \\
\cline { 2 - 8 } \multicolumn{1}{c|}{} & LJ11 & LM11 & LJ13 & LM13 & LJ17 & LM17 & Multiple Sensor \\
\hline $1^{\text {st }}$ & $5.3(\mathrm{~Hz})$ & 5.3 & - & 5.1 & 5.3 & 5.2 & 5.3 \\
Mode & 0.045 & 0.035 & - & 0.005 & 0.05 & 0.055 & 0.045 \\
\hline $2^{\text {nd }}$ & $6.7(\mathrm{~Hz})$ & 6.5 & 6.9 & 6.6 & 6.8 & 6.8 & 6.7 \\
Mode & 0.02 & 0.01 & 0.02 & 0.015 & 0.02 & 0.015 & 0.02 \\
\hline
\end{tabular}

The method identifies a bending mode at $5.3 \mathrm{~Hz}$ and a torsional mode at $6.7 \mathrm{~Hz}$. In this case the first torsion sensor (LJ13) was placed close to a node of the bending mode, and was therefore incapable of detecting it. It is worth remarking that the results obtained for individual sensors agree well among themselves and also show a good correlation with the multiple-sensor estimate. Due to their specific placement, a better measurement of the bending motion is of course expected from the flexion sensors (LJ11 \& LM11). On the other hand, the torsional response is best captured by LJ13 through LM17. Nevertheless, the two types of sensors are able to detect with reasonable accuracy both modes (with the exception of LJ13 which, as explained, lies too close to a node of the bending mode).

\section{Conclusions}

A method based in SVD and QR factorization with column pivoting has been presented. Timeshifting of the signal yields a symmetric square matrix from which noise is removed through SVD. In the process, the modal order of the system is also determined. Next, using QR factorization, a base of the space spanned by the modal vectors is built. Finally, SVD is utilized for modal parameters identification using a family of sinusoidal exponentially-damped functions.

The method presented overcomes the signal identification limitations found when testing flight conditions near flutter. In these cases, due to the proximity of adjacent modes and the short sample length, standard frequency domain-based methods suffer from severely degraded accuracy. The method described was applied to flutter flight-test data gathered during the IRIS-T / EF-18 integration campaign. The technique demonstrated adequate modal identification performance (in terms of damping and frequency).

Further studies to evaluate the impact in accuracy of non-linearity, sampling frequency, signal length, noise content and relative amplitude and damping of neighbouring modes are underway. Additional work is also needed in order to develop error-estimation criteria appropriate for the new method.

\section{Final consideration}

This paper is based on the research work carried out by the first author ( $\mathrm{J}$ Barros) under the supervision of Prof Lopez-Diez, his PhD Thesis advisor, and the collaboration of Dr Flores. After Prof Lopez-Diez's sudden death, in January 2013, Prof Martinez-Val has taken the responsibilities of PhD advisor. The authors dedicate this paper to the memory of the late Prof Lopez Diez, kept as third author as it was initially planned. 


\section{Bibliography}

[1] Kijewski TL and Kareem A. Wavelelet Transforms for System Identification in Civil Engineering. Computer-Aided Civil and Infrastructure Engineering 2003; 18, 339-355.

[2] Kijewski TL and Kareem A. On the presence of end effects and their melioration in wavelet-based analysis. Journal of Sound and Vibration 2002; 256, 980-988.

[3] Slavic J and Boltezar M. Damping Identification with the Morlet-Wave. Mechanical Systems and Signal Processing 2011; 25, 1632-1645.

[4] Slavic J, Simonovski I and Boltezar M. Damping identification using a continuous wavelet transform: application to real data. Journal of Sound and Vibration 2003; 262, 291-307.

[5] Slavic J and Boltezar M. Enhancements to the continuous wavelet transform for damping identifications on short signals. Mechanical Systems and Signal Processing 2004; 18, 10651076.

[6] Brenner MJ. Wavelet Analyses of F/A-18 Aeroelastic and Aeroservoelastic Flight Test Data. NASA Technical Memorandum 4793, April 1997.

[7] Brenner MJ. Non-Stationary Dynamics Data Analysis with Wavelet-SVD Filtering. Mechanical Systems and Signal Processing 17 (2003) 765-786.

[8] Le TH and Tamura Y. Modal Identification of Ambient Vibration Structure using Frequency Domain Decomposition and Wavelet Transform, Proc. 7th Asia-Pacific Conference on Wind Engineering, Taipei, Taiwan, November 8-12, 2009.

[9] Brenner and Pracenica C. Aeroelastic Flight Data Analysis with the Hilbert-Huang Algorithm. NASA Technical Memorandum 2006-213665, January 2006.

[10] Kejewski T and Kareem A. Reliability of Random Decrement Techniques for Estimates of Structural Damping. Proc. 8th ASCE Speciality Conference on Probabilistic Mechanics and Structural Reliability, PMC2000, South Bend, Indiana, Paper PMC2000-294.

[11] Rodrigues J and Brincker R. Application of the Random Decrement Technique in Aperational Modal Analysis. Proc. 1st International Operational Modal Analysis Conference, Copenhagen, Denmark, April 26-27, 2005.

[12] Kanjilal PP. Adaptive Prediction and Predictive Control. London, United Kingdom: Peter Peregrinus Ltd, 1995.

[13] Robert JB and Spanos PD. Random Vibration and Statistical Linearization. New York: Dover Publications, Inc, 1999.

[14] Kerschen G and Golinval JC Physical Interpretation of the Proper Orthogonal Modes Using the Singular Value Decomposition. Journal of Sound and Vibration 2002; 249, 849-865.

[15] Hong YP and Pan CT. Rank-Revealing QR Factorizations and the Singular Value Decomposition. Mathematics of Computation 1992; 58, 213-232.

[16] Juricek BC, Serborg DE and Larimore WE. Fault Detection using Canonical Variate Analysis. American Chemical Society 2004; 43, 458-474.

[17] Ewins DJ. Modal Testing. Second Edition. Baldock, United Kingdom; Research Studies Press Ltd, 2000. 
[18] An Eigensystem Realization Algorithm (ERA) for Modal Parameter Identification and Model Reduction - Juang and Pappa, Proc. of the Workshop on Identification and Control of Flexible Space Structures, Vol. 3: 299-318, April 1985.

[19] Gawronski K., Advanced structural dynamics and active control of structures, United States of America; Springer-Verlag New York, Inc., 2004.

[20] Changkyu Lee, Sang Wook Choi and In-Beum Lee, Adaptive monitoring statistics based on state space, 16th European Symposium on Computer Aided Process Engineering: 15451550, 2006.

[21] Berndt P, Kevin J and Alistair M, Modelling the dynamics of nonlinear time series using canonical variate analysis, Physica, D 170: 103-117, 2002. 


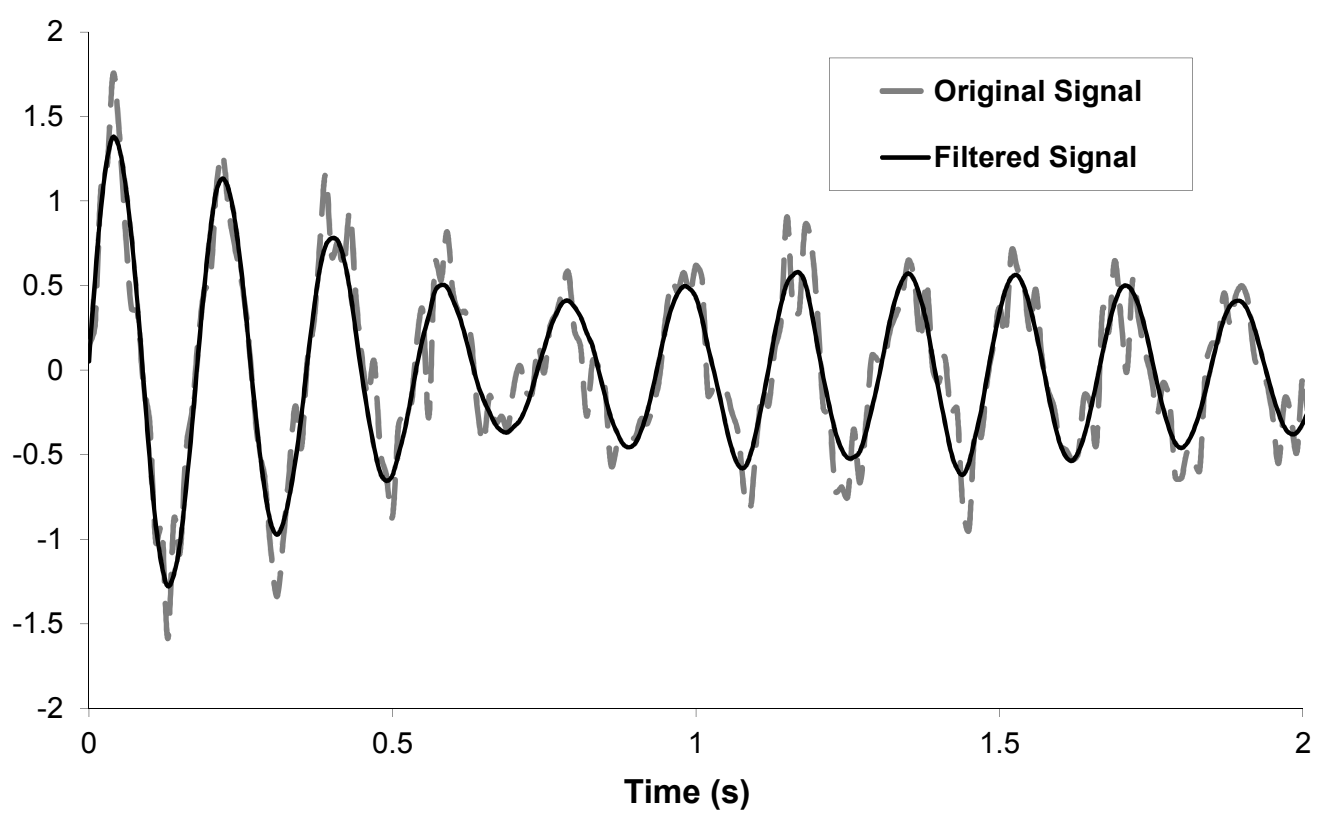

Figure 1 - Original signal and filtered data

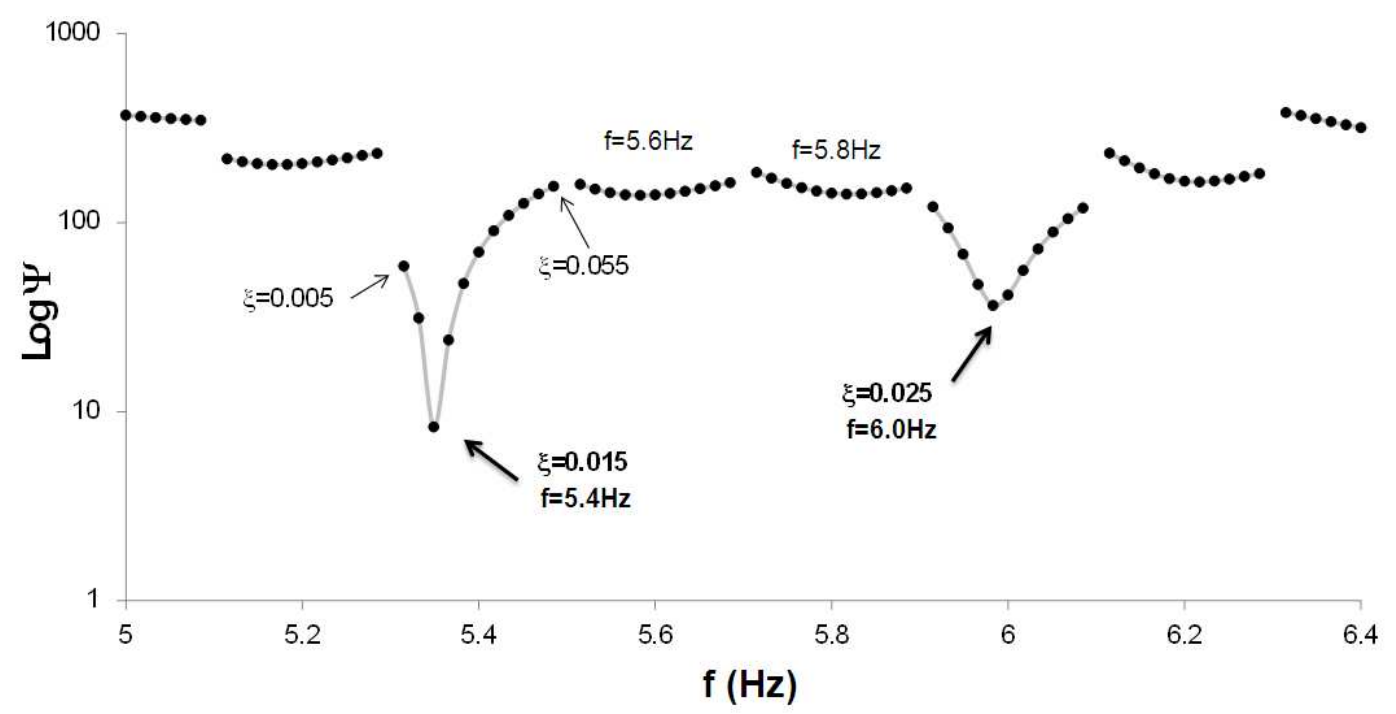

Figure 2 - Evolution of the $\Psi_{K}$ parameter as a function of the $\mathrm{f}_{\mathrm{K}}, \xi_{\mathrm{K}}$ combination 

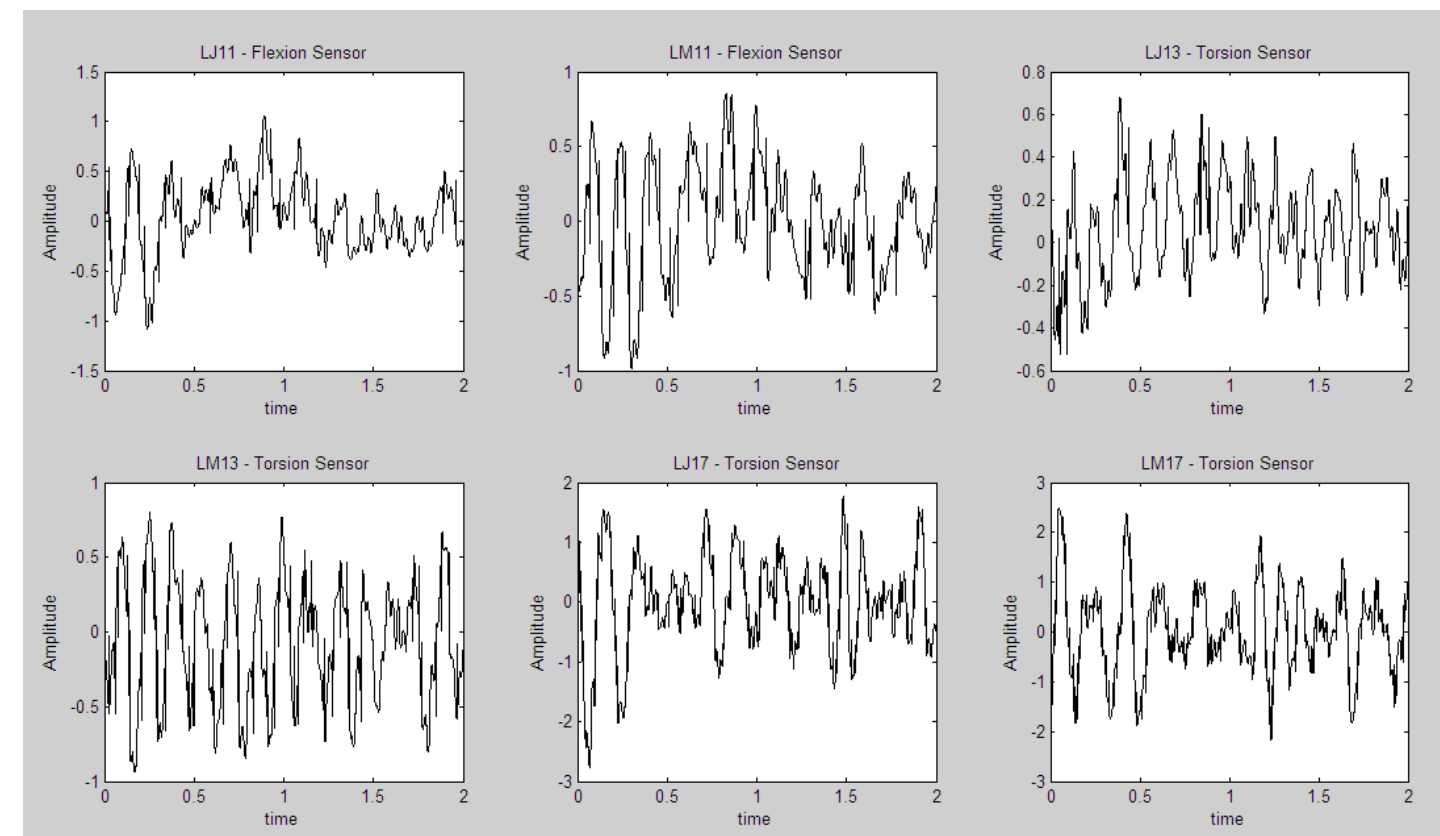

Figure 3- Raw signals from six different sensors
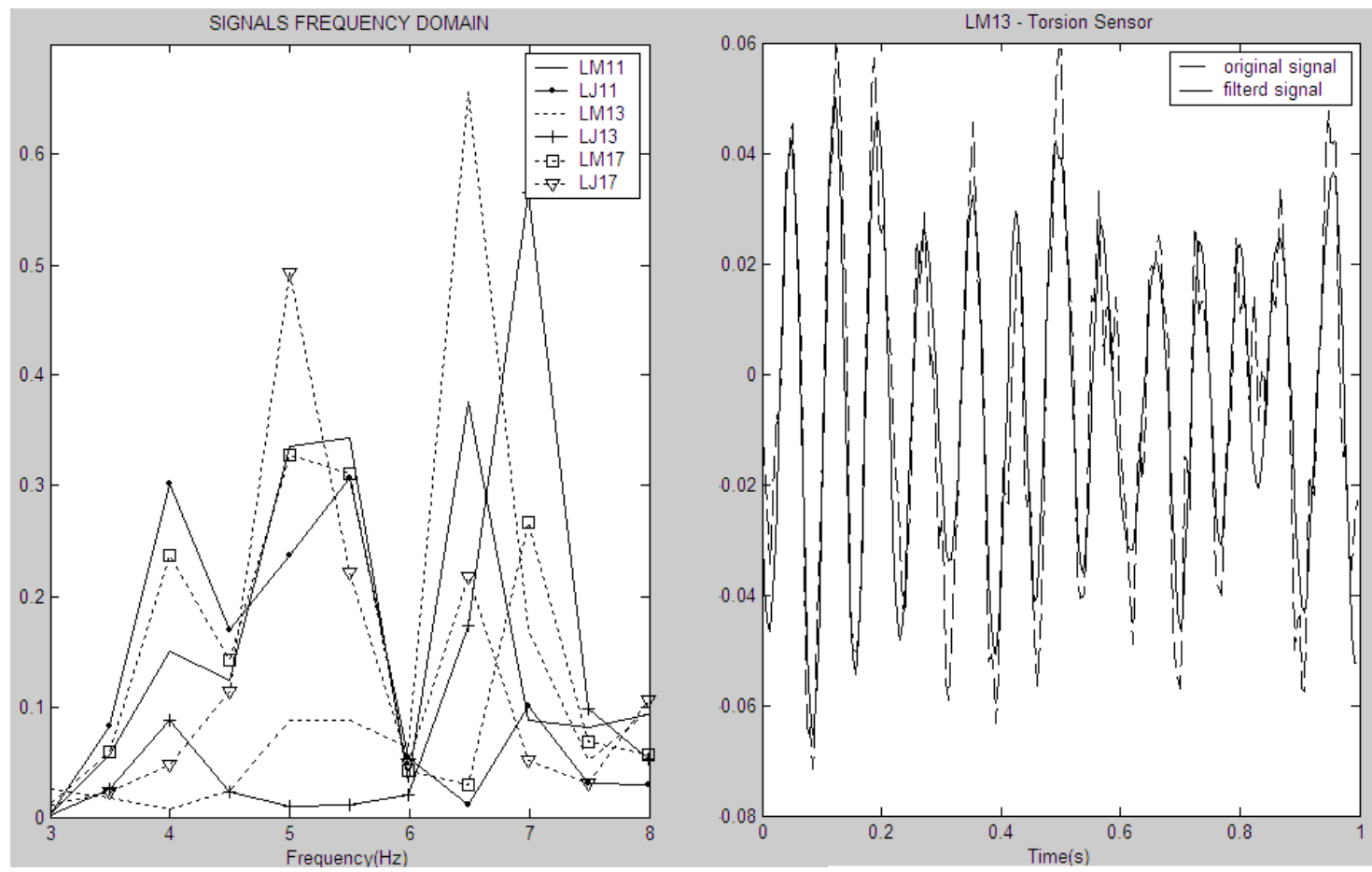

Figure 4- Signals Frequency Spectrum and LM13 Processed Signal 